\title{
Financial Transmission Rights (FTR) as a Congestion Management Scheme in Electricity Transmission: Strategic Behavior in a Coupled FTR - Electricity Market Model
}

\section{Sertac Oruc}

Technology Policy and Management Faculty, Delft University of Technology, Netherlands

\begin{abstract}
With the emergence of liberalized markets, transmission line congestion has been a prominent technical constraint that has to be accounted for in designing the new markets. Transmission line congestion is a phenomenon in electricity markets that emerges more severely in time with ever increasing demand in electricity and resultant excessive loading of transmission lines. Various congestion management techniques such as market splitting, market coupling etc. are utilized currently in energy markets. However on the long run financial transmission rights (FTR), which is readily used by some US electricity market operators such as PJM, New York and New England operators, has potential to dominate other markets too. Modelling financial transmission rights to test some strategic hypotheses is imperative to be able to introduce suitable policies and regulations. In this paper we present a modelling approach for financial transmission rights and examine strategic use of hidden knowledge in a hypothetical electricity - FTR market.
\end{abstract}

Keywords: Finance, Institutions, Transmission Rights

\section{SUBHEAD REQUIRED}

Electric industry has been going through fundamental institutional changes by constantly moving away from an integrated centralized regulated system towards a market based decentralized self-organizing industry. There remains various mechanism design questions that would ideally guide the system towards a state where certain technical and socio-economic constraints are not breached.

With the emergence of liberalized markets, transmission line congestion has been a prominent technical constraint that has to be accounted for in designing the new markets. Transmission line congestion is a phenomenon in electricity markets that emerges more severely in time with ever increasing demand in electricity and resultant excessive loading of transmission lines. Various congestion management techniques such as market splitting, market coupling etc. are utilized currently in energy markets. However on the long run financial transmission rights (FTR), which is readily used by some US electricity market operators such as PJM, New York and New England operators, is expected to dominate. Additionally with the emergence of a need for a pan-European grid, FTR is being increasingly considered as a Europe wise mechanism to tackle congestion management problem. Modeling financial transmission rights to test some strategic hypotheses regarding this emerging market in Europe is crucial to be able to introduce suitable policies and regulation rule. Hence in this working paper we present a modeling approach for financial transmission rights markets for testing strategic hypotheses. A research agenda, which utilizes the mentioned approach, is set.

Every networked infrastructure needs to be treated in light of their particular technical functionalities as well as 
associated social and political acceptances. The electricity system is not different in this regard, given its broad application field that affect three quarters of people on earth ${ }^{11}$ and its very complex technical specifications. These specifications include but are not limited to storage problems, requirement of market clearance and loop flows. Safeguarding these critical technical specifications, which emerge from the very nature of these industries, is an obligation. In some situations strategic behavior exercised by the competitive companies of the deregulated markets may hinder the delivery of the critical service ${ }^{2}$.

Congestion in transmission lines is a particular phenomenon to be considered in this regard ${ }^{3}$. Thermal limits, voltage stability and voltage drop regulations impose transmission capacity limitations on transmission lines. Thus distribution of electricity flows among the transmission lines should be regulated in order not to exceed these transmission capacity limits. Owing to this fact, many congestion management methods have been devised in the literature ${ }^{4}$ and many of them are actually utilized in various electricity markets ${ }^{5}$

Electric power transmission systems are utilized to transfer bulk electricity from generators to loads. The transmission occurs on the transmission networks or power grids, which is comprised of high voltage transmission lines. These transmission lines cannot carry beyond a certain limit depending on the heat related properties of the cable. Electricity transmission congestion refers to the situation when this limit is reached and no marginal megawatts can be pushed into the transmission network. The effects of congestion over a line can jeopardize the security of the entire system. A higher quantity of permitted flow through the line might overheat it, causing the elongation of the material which in some cases can be permanent. This situation enhances the probability of failure or even blackout with severe social and economic consequences ${ }^{6}$.

According to general equilibrium theory, a market is called perfect if information is transparent, no barriers of entry or exit exist, no participant abuses market power to set prices and equal access to production technology is provided ${ }^{7}$. Projection of this view of perfect market would mean that an ideal market for electricity must provide a platform that is spatially homogeneous in terms of pricing, in order to allow for entrance and exit of traded power with the same price. However since electricity flows according to Kirchhoff's laws, and the flow can be constrained by cable flow capacities, the free flow of traded power may be hindered. This is usually overlooked by the scholars who employ a purely economic perspective. Instead of utilizing the cheapest power, more expensive power has to be utilized in order to satisfy the demand due to transmission capacity constraints. As a result some price differences between the nodes occur. This is frequently referred to as congestion cost. The different prices, caused by spatial heterogeneity, are called locational marginal prices $(\mathrm{LMP})$ or nodal prices in the literature.

The management of congestion implies different results for the efficiency of the generation market. Hence different mechanisms for congestion management have been suggested to allocate scarce transmission and to maintain a competitive market. Some methods are more focused on preventive measures, since they try to avoid the congestion beforehand, while others attempt to solve the congestion in real time. The methods used are dependent on the evolution of the institutional setting within the respective region because of different pace of development in different parts of Europe. Some points must be taken into account while considering market mechanisms for congestion management. First of all, it should be noted that the transmission operator is a natural monopoly and it always has a pivotal position. The network operator cannot be relied upon without regulation to secure the public interest. The regulation applied to TSO is crucial with respect to market power concerns. Secondly it should be realized that the supply and demand varies instantaneously while the market clearance must occur at all times. This may result in volatility since the demand as well as the supply might be volatile due to many factors. Thirdly it is important to note that the configuration of the physical network causes network externalities called loop flows. Transportation of electricity does not occur as in the case of a transportation of a physical good in a classical market. Electricity flows through the least

1 Gronewold, N. One-quarter of world's population lacks electricity. Scientific American (2009).

2 Borenstein, S., Bushnell, J. \& Knittel, C. Market power in electricity markets: Beyond concentration measures. Energy Journal 20, 65-88 (1999).

3 Knops, A. \& Vries, D. Congestion management in the European electricity system: an evaluation of the alternatives. Competition and Regulation in Network Industries 2, 311-352 (2011).

4 Kumar, A., Srivastava, S. \& Singh, S. Congestion management in competitive power market: A bibliographical survey. Electric Power Systems Research 76, 153-164 (2005).

5 De Vries, L. \& Hakvoort, R. The question of generation adequacy in liberalised electricity markets. Delft University of Technology (2004).

6 Fang, R. S. \& David, A. K. Transmission congestion management in an electricity market. IEEE Transactions on Power Systems 14, 877-883 (1999).

7 Debreu, G. Theory of value: An axiomatic analysis of economic equilibrium. (Yale Univ Pr, 1959). 
resistant cables from high voltage to low voltage level. So, in a hypothetical three node network, sending power from node $\mathrm{A}$ to node $\mathrm{B}$ is always affected by the resistance of the lines including those involving node $\mathrm{C}$.

\section{LOCATIONAL MARGINAL PRICING (LMP) AND FINANCIAL TRANSMISSION RIGHTS (FTR)}

LMP is spatial pricing of electricity in which different price zones are formed based on occurrences of transmission congestion on the electricity transmission lines. The electricity prices resulting at each node are called locational marginal prices. In a fully connected network, LMPs on different nodes may end up with different values even if only one transmission line is congested throughout the network. Due to Kirchoff laws, injection and withdrawal of electricity on any node possibly affects the electricity load on any transmission line ${ }^{8-11}$.

FTR is a hedging tool against congestion charges for markets that are based on locational marginal pricing (LMP). Thus LMP or nodal pricing is the prime mechanism on which FTR is built on and has been applied in the electricity networks of New York, PJM and New England regions in the US ${ }^{9}$. Since LMP is the underlying mechanism that brings about the need for FTRs, in this sections we further elaborate on LMP together with FTR. Then we will explain the functionality of FTRs and finally test the strategy-proneness of FTRs in a game theoretical model.

With FTR model, the TSO computes the LMP for each congestion-prone node, which carries the associated congestion costs. This would result in volatile prices in electricity as the congested lines fragment the markets spatially into minor regions. To hedge these volatilities the generation companies can buy FTRs offered by the TSO's. The FTRs entitle the holder to receive the price difference between two stated nodes on the FTR. FTRs are funded by the congestion rent collected by the TSO.

FTRs are utilized as a hedge against the congestion costs due to different LMPs on the generation and consumption nodes. The price differences across the nodes may result in unexpected costs due to the foreseen or unforeseen congestion occurrences.

\section{A GAME THEORETICAL MODEL OF COUPLED FTR-ELECTRICITY MARKET}

The model proposed in this research is a coupled electricity and FTR market model. It features both FTR market and the electricity market which are coupled by the decisions of the electricity firms and the price signals that affect each market recursively. Most of the models readily available in the literature are based on assumed LMPs, thus ignore electricity market part of the mechanism or based on stochastic FTR values in conjunction with the electricity equilibrium models. However two distinct market structures, the signals of which affect each other is a more accurate view of the total system. This is the perspective that we employ in this research.

One specific strategic hypothesis that we test in this analysis can be formulated as follows: "Can firms exercise market power in electricity market by keeping their generation information private?" This question requires us to consider market power of the firms in the electricity market and amount of FTRs it can acquire. Moreover two markets are interrelated as the evaluation of FTRs depend on the results of electricity market. To test strategic hypotheses such as the one stated here, one needs to model electricity market as well as the FTR market.

In order to assess the strategy-proneness of FTR method, we use our coupled FTR - electricity market model that is briefly described above. The model simulates a scenario of strategic behavior based on hidden information. The scenario clearly shows that hiding private information, which is about a generation technology upgrade in this case, can give a generation company or any other market participant tremendous market edge, making FTR markets quite information sensitive. The information sensitiveness clearly serve to the incumbent firms, which has larger presence in the generation market and hence more opportunity to hoard information. As a result introducing FTRs without a mechanism to protect small players from information hoarding would contradict with the goal of creating a perfect market with low barrier of entry and high number of players.

\footnotetext{
8 Green, R. J. \& Newbery, D. M. Competition in the British electricity spot market. Journal of political economy, 929-953 (1992).

10 Oruç, S. \& Cunningham, S. W. 1-6 (IEEE).

11 Oruç, S. \& Cunningham, S. W. Transmission Rights to the Electrical Transmission Grid in the Post Liberalization Era. Journal of the Knowledge Economy, 1-20, doi:10.1007/s13132-012-0130-7 (2012)

9 Hogan, W. W. Contract networks for electric power transmission. Journal of Regulatory Economics 4, 211-242 (1992).
} 
As far as the policymaking is concerned, the main takeaway from this study is that FTR markets can give further opportunity for the market participants in the electricity financial transmission rights market. FTR could be an appropriate mechanism for the generators to hedge their congestion risk. However the increasing complexity of the total electricity system would render the system more prone to strategic behavior. In addition to the strategic behavior by creating congestion that is discussed in the literature, we discussed another strategic behavior by hidden knowledge in this study. On the other hand the other congestion management methods have their respective disadvantages as well as advantages with respect to the FTR scheme that we analyzed in this study. A comparison of different schemes falls out of the scope of this study and can be treated as a future extension.

As for possible remedies against strategic behavior based on hidden knowledge, policy makers might consider looking at the rules and regulations of the stock markets as some analogy between the stock markets and FTR markets can be drawn as long as the hidden knowledge is concerned. To our knowledge, insider trading is forbidden in stock exchanges and the stock mobility of the executives of the companies are heavily regulated. Some similar regulations regarding the FTR movements of the generation companies can also be implemented in FTR markets. 